\title{
RETARDANTES DE CHAMA BROMADOS: UMA REVISÃO
}

\author{
Michelle C. Pieronia , Juliana Leonel ${ }^{\mathrm{b}, *}$ e Gilberto Fillmanna \\ anstituto de Oceanografia, Universidade Federal do Rio Grande, 96203-900 Rio Grande - RS, Brasil \\ ${ }^{b}$ Departamento de Oceanografia, Instituto de Geociências, Universidade Federal da Bahia, 40170-020 Salvador - BA, Brasil
}

Recebido em 23/05/2016; aceito em 09/08/2016; publicado na web em 29/09/2016

\begin{abstract}
BROMINATED FLAME RETARDANTS: A REVIEW. Polybrominated diphenyl ethers (PBDEs), Polybrominated biphenyls (PBBs), Hexabromocyclododecane (HBCD) and Tetrabromobisphenol A (TBBP-A) are flame retardant widely used in plastics, textiles, furniture, consumer electronics, automobiles, among others. They are important components added to retard the spread of flame and, thus, mitigate deaths and destruction caused by fires. However, environmental studies conducted in several places show that these compounds exhibit toxic effects. In addition, some are resistant to degradation, persistant in the environment, bioaccumulate and biomagnify in biota, which have worried the scientific community and government agencies. Therefore, production and use of some brominated flame retardants is being restricted and / or prohibited in many countries and some have recently been listed in the Annexs of Stockholm Convention as Persistent Organic Pollutants (POPs). Thus, a review of their use, production, legislation and environmental occurrence is presented, as well as a brief introduction to the new generation of flame retardants.
\end{abstract}

Keywords: Persistent Organic Pollutants (POPs); Polybrominated biphenyls (PBBs); Polybrominated diphenyl ethers (PBDEs); Tetrabromobisphenol A (TBBP-A); Hexabromocyclododecane (HBCDs).

\section{INTRODUÇÃO}

O crescimento populacional e o consequente aumento da produção de novos materiais levaram à geração e utilização de uma ampla variedade de polímeros sintéticos, empregados nos mais variados bens de consumo, indústrias e construção civil. A maioria destes polímeros é constituída à base de derivados de petróleo, portanto inflamáveis, havendo assim a necessidade de promover medidas que retardem a propagação das chamas. ${ }^{1}$

O uso de retardantes de chama data de 450 A.C. por egípcios, que utilizavam alume para diminuir a inflamabilidade da madeira; mais tarde, em 200 A.C., os romanos passaram a utilizar uma mistura de alume e vinagre para o mesmo fim. Foi, porém, a partir dos anos 1970, com o aumento do consumo de materiais poliméricos, que houve uma necessidade ainda maior de prover segurança contra incêndios, sendo desenvolvidos, para tanto, novos retardantes de chamas adequados a estes polímeros. ${ }^{2}$ Atualmente existem mais de 175 produtos químicos classificados como retardantes de chama, divididos em quatro grandes grupos: a) inorgânicos (hidróxidos de alumínio, antimônio e estanho); b) orgânicos halogenados (clorados e bromados); c) organofosforados e d) à base de nitrogênio. Cerca de $40,4 \%$ da sua produção mundial correspondendo a hidróxido de alumínio, $19,7 \%$ de bromados, $14,6 \%$ de fosforados, $11,3 \%$ de clorados, $8,4 \%$ de óxido de antimônio e 5,6\% de outros. ${ }^{2,3}$ Em 2011 a produção mundial de retardantes de chamas foi de 1,9 milhões de toneladas. ${ }^{4}$

Esses compostos podem retardar a propagação das chamas por meio de ações físicas ou químicas. No caso das físicas, agem por meio de resfriamento quando os compostos liberam partículas de água ao serem expostos ao calor, ou pela formação de uma camada protetora que envolve os materiais. Já nas ações químicas, na presença de calor os retardantes reagem com o ar, produzindo gases incombustíveis que reduzem o suprimento de oxigênio que seria utilizado pela combustão ou, ainda, inibem a combustão ao reagir com os polímeros na fase sólida, formando uma camada protetora rica em carbono. ${ }^{5}$

Existem mais de 75 diferentes compostos utilizados como

*e-mail: juoceano@gmail.com retardantes de chama bromados (BFRs). ${ }^{1}$ Segundo o Bromine Science and Environmental Forum, em 2000 houve uma demanda global de mais de 310.000 toneladas de BFRs. ${ }^{6}$ Esses retardantes estão entre os mais usados devido ao seu baixo custo, alta eficiência contra a propagação de chamas e fórmulas adaptáveis a quase todos os polímeros. ${ }^{1}$ Os BFRs são divididos em três subgrupos, dependendo do modo de incorporação em polímeros: monômeros (estirenos bromados e butadieno bromados), reativos (tetrabromobisfenol A) e aditivos (bifenilas polibromadas, éter difenílicos polibromados, hexabromociclododecano e tetrabromobisfenol A). Os monômeros bromados são usados na produção de polímeros bromados, os reativos são ligados quimicamente ao polímero e os aditivos são apenas misturados aos polímeros. Em razão disso, os aditivos são mais propensos a serem lixiviados para o ambiente. ${ }^{1}$

Os retardantes de chama bromados reativos e aditivos, ou seja, as bifenilas polibromadas (PBBs), os éter difenílicos polibromados (PBDEs), o tetrabromobisfenol A (TBBP-A) e os isômeros de hexabromociclododecano (HBCDs) têm atraído grande atenção na última década devido a sua grande produção e amplo uso, bem como pela sua constante detecção no meio ambiente e nos seres humanos. ${ }^{1,7-10}$ Os retardantes de chamas bromados são, de maneira geral, persistentes, lipofílicos e apresentam elevado potencial para bioacumulação, tendo sua ocorrência na biota associada a efeitos deletérios, além de produzirem grande quantidade de fumaça e gases tóxicos nos incêndios. ${ }^{11,12} \mathrm{~A}$ presença desses compostos já foi relatada em todos os compartimentos ambientais e em diferentes regiões do globo, incluindo regiões remotas onde não há histórico do seu uso e/ou produção (por exemplo, as regiões polares). ${ }^{13-15}$

Alguns desses compostos (PBBs, Penta-BDE, Octa-BDE e DecaBDE) já tiveram a sua produção e comercialização restringida ou proibida em alguns países (ex. União Europeia e Japão). Em 2009, os tetrabromodifenis éter (tetra-BDE), os pentabromodifenis éter (penta-BDE), os hexabromodifenis éter (hexa-BDE), os heptabromodifenis éter (hepta-BDE) e o hexabromobifenil (HxBB) foram incluídos no Anexo A da Convenção de Estocolmo. Por serem considerados Poluentes Orgânicos Persistentes (POPs) tiveram a sua produção, uso e comercialização proibida, visando a sua total eliminação. ${ }^{16} \mathrm{O}$ 
hexabromociclododecano (HBCD) foi incluido no Anexo A em 2013. Já as misturas comerciais hexa-, octa- e decabromobifenil (PBB) e penta- e octabromodifenil éter (BDEs) foram incluídas no Anexo III da Convenção de Roterdã em 2008 e 2013, respectivamente. ${ }^{17}$ Esta Convenção promove a responsabilidade compartilhada e o consentimento informado prévio no comércio internacional das substâncias químicas consideradas perigosas, em um esforço cooperativo entre as partes, visando a proteção da saúde humana e do meio ambiente.

No Brasil, ainda não há uma legislação específica em relação a estes retardantes, apenas um Projeto de Lei do Senado $\left(n^{\circ} 173\right)$ proposto em 2009 e arquivado em 2011, que estabelece que computadores, componentes de computadores e equipamentos de informática em geral comercializados no Brasil devem apresentar concentração não superior a $0,1 \%$ de PBBs, PBDEs, chumbo, mercúrio e cromo hexavalente. ${ }^{18}$ Além disso, há apenas uma instrução normativa (número 1 de 2010) a respeito dos critérios ambientais na aquisição de bens, contratação de serviços ou obras por orgãos públicos, afirmando que estes poderão exigir, entre outros que: "os bens não contenham substâncias perigosas em concentração acima da recomendada na diretiva RoHS (Restriction of Certain Hazardous Substances), tais como mercúrio (Hg), chumbo $(\mathrm{Pb})$, cromo hexavalente $(\mathrm{Cr}(\mathrm{VI}))$, cádmio $(\mathrm{Cd})$, bifenil-polibromados (PBBs), éteres difenil-polibromados (PBDEs)" ${ }^{19}$

\section{BIFENILAS POLIBROMADAS (PBBs)}

Bifenilas polibromadas (PBBs) (Figura 1) são um grupo de retardantes de chama que começaram a ser fabricados no início da década de 1970 e mesmo durante o período de sua máxima utilização não chegaram a representar $1 \%$ das vendas mundiais de retardantes de chama. ${ }^{20}$ Sua aplicação se limitou praticamente a termoplásticos (acrilonitrila-butadieno-estireno - ABS) utilizados em equipamentos eletrônicos de uso doméstico e empresarial. ${ }^{20,21}$ No Brasil não foram identificados dados sobre o uso do PBBs no passado, nem de sua produção ou existência de estoques. ${ }^{22,23}$ Da mesma forma, não foram feitas importações ou exportações destes compostos no período de 1997 a 2015, não existindo dados para os anos anteriores. ${ }^{24}$

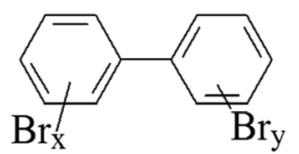

Figura 1. Estrutura química genérica da Bifenila Polibromada (PBB), onde $x+y=1-10$

Os PBBs fazem parte de uma classe de compostos que podem ter de um a dez átomos de hidrogênio substituídos por bromo. Dessa for$\mathrm{ma}$, apresentam estrutura semelhante às bifenilas policloradas (PCBs) com uma nomeclatura análoga e um mesmo número de congêneres. ${ }^{25}$ Sua produção comercial, entretanto, envolve um número restrito de compostos onde apenas 42 congêneres de PBBs foram sintetizados em laboratório. ${ }^{26}$ Eles foram disponibilizados na forma de três misturas comerciais (NTP 2011): a) Hexabromobifenil (HxBB) - formado por 4,0\% de pentabromobifenis, $62,6 \%$ de hexabromobifenis e $33,4 \%$ de heptabromobifenis. Foi a principal mistura produzida representando até $87 \%$ da produção de PBBs, sendo desses $98 \%$ produzidos na forma do produto comercial conhecido como FireMaster BP 6; b) Octabromobifenil (OBB) - sua composição possui 1,8\% de heptabromobifenis, $47,3 \%$ de nonabromobifenil, $45,2 \%$ de octabromibifenis e 5,7\% de decabromobifenil; c) Decabromobifenil (DBB) - mistura de $96,8 \%$ de decabromobifenil, $2,9 \%$ de nonabromobifenil e $0,3 \%$ octabromobifenil. ${ }^{21}$

Esses compostos, principalmente as misturas $\mathrm{HxBB}$ e o $\mathrm{OBB}$, foram produzidos nos Estados Unidos pela Michigan Chemical Corporation; na Grã-bretanha duas empresas foram responsáveis pela produção do DBB; na Alemanha fabricou-se o Bromkal 80-9D (basicamente nonabromobifenil); na França a empresa Atochem comercializou o DBB. ${ }^{27}$ Por terem estrutura semelhante, apresentam propriedades físico-químicas similares às bifenilas policloradas (PCBs) (Tabela 1), sendo também tóxicas, resistentes à degradação, hidrofóbicos, lipofílicos e, portanto, bioacumulativos. ${ }^{28}$ Devido a estas características, a mistura comercial hexabromobifenil foi incluída na lista dos POPs em maio de 2009. ${ }^{9}$ Apesar da semelhança com os PCBs, a ocorrência dos PBBs no ambiente é mais limitada devido a sua baixa produção e menor tendência de liberação a partir dos termoplásticos nos quais foram incorporados..$^{29} \mathrm{Os} \mathrm{PBBs}$ ganharam bastante atenção após um acidente em Michigan (EUA), entre 1973 e 1974, quando cerca de 250-500 quilos de FireMaster BP-6 (HxBB) foram acidentalmente adicionados à ração animal, sendo a população local posteriormente exposta através da ingestão de leites e outros alimentos contaminados. ${ }^{20,30}$ Como consequência do acidente, a empresa parou de produzir FireMaster BP-6 em 1974 e demais PBBs em 1977.20,31

Apesar da aplicação dos PBBs ter diminuído desde meados da década de 1970, resíduos dos mesmos continuam sendo reportados em amostras ambientais e em seres humanos de diferentes partes do globo, como China, Espanha e Estados Unidos. ${ }^{25,33-37}$ Os congêneres mais comumente encontrados são o PBB 153 e outros de baixa bromação como o PBB 29, 49, 52, 56, 77 e 80..$^{34,35,38,39}$

Resíduos de PBBs foram detectados em compartimentos ambientais como lodo de tratamento de efluentes domésticos na Austrália e em amostras de diversas espécies de peixes de valor comercial da Polônia e França, do Mar Báltico e do nordeste dos EUA, em cetáceos da costa sul e sudeste do Brasil e do Atlântico Norte, aves e ar do Ártico e em pandas na China. ${ }^{15,33,34,39-42}$ Muitos trabalhos referem-se especialmente ao acidente ocorrido em Michigan ou a região de descarga de efluentes da Michigan Chemical Corporation. ${ }^{25,31,36,37,43}$ Entre eles, alguns reportaram valores elevados em amostras de sangue humano (933 a $\left.1744 \mu \mathrm{g} \mathrm{L}^{-1}\right)$ e em amostras de água $\left(0,01\right.$ e $\left.0,07 \mu \mathrm{g} \mathrm{L}^{-1}\right)$ e sedimento (100 e $\left.77000 \mathrm{ng} \mathrm{g}^{-1}\right){ }^{25,31}$

A absorção dos PBBs pelos organismos ocorre de forma rápida e inversamente proporcional ao número de bromos na molécula. ${ }^{44}$ Devido a sua característica de lipofilicidade, as maiores concentrações são encontradas, principalmente, nos tecidos hepático e adiposo. ${ }^{44,45}$

Tabela 1. Propriedades físico-químicas das séries homólogas mais relevantes de $\mathrm{PBB}^{32}$

\begin{tabular}{|c|c|c|c|}
\hline \multirow{2}{*}{ Propriedades } & \multicolumn{3}{|c|}{ PBBs } \\
\hline & НехаBВ & OctaBB & DecaBB \\
\hline Formula molecular & $\mathrm{C}_{12} \mathrm{H}_{4} \mathrm{Br}_{6}$ & $\mathrm{C}_{12} \mathrm{H}_{2} \mathrm{Br}_{8}$ & $\mathrm{C}_{12} \mathrm{Br}_{10}$ \\
\hline Pressão de vapor & $5,2 \times 10^{-8} \mathrm{~mm} \mathrm{Hg}$ a $25^{\circ} \mathrm{C}$ & $7,1 \times 10^{-11} \mathrm{~mm} \mathrm{Hg}$ a $28^{\circ} \mathrm{C}$ & - \\
\hline Solubilidade em água & $11 \mu \mathrm{g} \mathrm{L}^{-1}$ a $25^{\circ} \mathrm{C}$ & 20 a $30 \mu \mathrm{g} \mathrm{L}^{-1}$ a $25^{\circ} \mathrm{C}$ & Insolúvel \\
\hline $\log \mathrm{K}_{\mathrm{OW}}$ & 6,39 & 5,53 & 8,58 \\
\hline Constante da Lei de Henry & - & $3,9 \times 10^{-6} \mathrm{~atm}-\mathrm{m}^{3} \mathrm{~mol}^{-1}$ & - \\
\hline
\end{tabular}


Além disso, estudos feitos com humanos mostraram que a transferência placentária ocorre, porém a principal forma de excreção em mamíferos é através do leite. ${ }^{46,47} \mathrm{~A}$ meia-vida desses compostos nos organismos é bastante variável, por exemplo, 22 dias em porquinhos-da-índia a 4 anos em macacos. ${ }^{48,49}$ Em humanos, pode variar de 8 a 30 anos. ${ }^{43}$ Esses tempos vão depender da idade, massa corpórea, gestação, histórico de vida, etc. Em sedimentos e solos a meia-vida é maior que 6 meses, devido à resistência de misturas de PBB à degradação microbiana. ${ }^{50}$

Embora não sejam claros todos os efeitos decorrentes da contaminação por PBBs, estudos feitos com ratos mostram déficit na função neuromuscular, na atividade locomotora, retardo de crescimento e toxicidade neuro comportamental. ${ }^{30}$ Os efeitos sentidos pela população de Michigan, afetada pelo acidente de 1973, incluem alterações gastrointestinais, cutâneas, músculo-esqueléticas e neurológicas. ${ }^{36,37,51}$ Outros estudos feitos ainda mostram relação entre a exposição aos PBBs e incidência de câncer, alteração de crescimento, e alteração no ciclo menstrual. ${ }^{44,45,52,53}$

Nos EUA, a produção do HxBB foi descontinuada em $1976,{ }^{20}$ mas a produção do OBB e DBB continuou até $1979 .{ }^{20,54} \mathrm{Na}$ Grã-Bretanha a produção dos PBBs foi descontinuada em 1977 e na Alemanha em 1985. ${ }^{21}$ No Brasil, segundo o Ministério do Meio Ambiente não foram identificados dados de produção e uso industrial do HxBB. ${ }^{22,23}$ Outros países, como Austrália, Japão e República do Líbano, também reportaram que não mais produzem ou usam o HxBB. ${ }^{27}$ Por fim, a França foi o último país a cessar a produção de PBBs em $2000 .{ }^{21}$ De acordo com a UNEP, devido à pequena produção e ao uso limitado dos $\mathrm{HxBB}$, acredita-se que a maioria dos materiais que os continha tenham sido eliminados. ${ }^{55}$ Ainda assim, os $\mathrm{HxBB}$ foram inseridos na lista de POPs da Convenção de Estocolmo em 2009.

\section{ÉTER DIFENÍLICOS POLIBROMADOS (PBDEs)}

Os PBDEs (Figura 2) começaram a ser produzidos no final da década de 1970 através da bromação do éter difenílico, na presença de um catalisador (por exemplo, $\mathrm{AlCl}_{3}$ ) em solvente. ${ }^{1,56} \mathrm{~A}$ substituição de qualquer um dos 10 átomos de hidrogênio do éter difenílico por bromo (Figura 2) possibilita a formação de até 209 congêneres de PBDEs. ${ }^{1}$

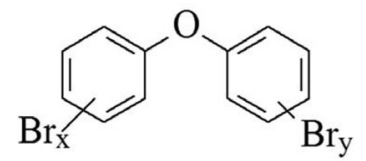

Figura 2. Estrutura química genérica do Éter Difenílico Polibromado (PBDE), onde $x+y=1-10$

Comercialmente os PBDEs foram produzidos principalmente na forma de três misturas técnicas: Penta-BDE, Octa-BDE e DecaBDE. ${ }^{56} \mathrm{~A}$ mistura penta-BDEs, composta principalmente por $24-37 \%$ de tetra-BDEs, $50-60 \%$ de penta-BDE, $4-8 \%$ de hexa-BDEs, foi empregada principalmente na manufatura de espumas flexíveis de poliuretano, utilizadas na confecção de cadeiras, poltronas, sofás, colchões, cortinas, entre outros. ${ }^{56,57}$ A mistura octa-BDE, composta principalmente por $10-12 \%$ de hexa-BDEs, $44 \%$ de hepta-BDEs, $31-35 \%$ de octa-BDEs, $10-11 \%$ de nona-BDEs e $<1 \%$ de deca-BDE foi aplicada na manufatura de polímeros à base de acrilonitrila-butadieno-estireno (ABS), usado em monitores de computador e em outros aparelhos eletrônicos correlatos. ${ }^{57,58}$ Por fim, a mistura deca-BDE, composta por $>97 \%$ de BDE 209 e o restante por nona-BDEs e resíduos de octa-BDEs, é empregada em têxteis e em plásticos de alta densidade usados em televisões, computadores e outros equipamentos eletrônicos..$^{57,59}$

Devido as suas propriedades (Tabela 2), os PBDEs são resistentes à degradação físico-química e biológica e, consequentemente, apresentam alta persistência no ambiente. São lipofílicos e hidrofóbicos, podendo então bioacumular e biomagnificar ao longo da cadeia trófica. Apresentam toxicidade aos organismos e, devido a sua baixa pressão de vapor, podem ser transportados a longas distâncias..$^{58,60}$ Essas características levaram os congêneres tetra- e penta-BDE, pertencentes à mistura técnica penta-BDE, e os congêneres hexa- $\mathrm{e}$ hepta-BDE componentes da mistura técnica octa-BDE, a serem incluídos na lista dos Poluentes Orgânicos Persistentes (POPs) da Convenção de Estocolmo em maio de 2009. ${ }^{9}$ Alguns congêneres de PBDEs, das misturas penta e octa-BDEs já haviam tido sua produção e comercialização proibida em alguns países. ${ }^{61} \mathrm{~A}$ União Europeia baniu as formulações comerciais penta- e octa-PBDE em 2003, porém a mistura deca-BDE continuou em uso. ${ }^{57}$ Nos EUA, as empresas decidiram voluntariamente parar a produção da formulação deca-BDE no final de 2013. ${ }^{62}$ Em outubro de 2013, a Noruega propôs à Convenção de Estocolmo que a formulação comercial deca-BDE também fosse incluída no Anexo A da Convenção de Estocolmo. ${ }^{63}$

Apesar da restrição atual de uso de alguns PBDEs, o aporte ambiental destes compostos ainda é motivo de preocupação. A restrição do uso não impede que os produtos fabricados anteriormente à proibição continuem sendo utilizados ou que o seu destino final, seja na reciclagem ou disposição dos bens de consumo em lixões, acabe consequentemente liberando, de forma lenta e difusa, o contaminante para o ambiente. ${ }^{61}$ Adicionalmente, como consequência da restrição das misturas penta- e octa-BDE, a produção de deca-BDE aumentou e, apesar deste ser considerado um congênere menos biodisponível, pode sofrer debrominação gerando formas mais tóxicas e capazes de bioacumular. ${ }^{61,65,66}$

Os PBDEs não são quimicamente ligados aos polímeros, podendo então ser separados da matriz polimérica durante algumas atividades como incineração de componentes eletro-eletrônicos e bens de consumo, ou deposição de lixos eletrônicos e transformadores em lixo comum, resultando na introdução desses compostos no ambiente..$^{56,67}$

Após sua entrada no ambiente, esses compostos são encontrados preferencialmente na biota e sedimento. Os níveis mais altos desses compostos encontram-se em região altamente industrializada e com maior densidade populacional, onde as concentrações apresentam-se

Tabela 2. Propriedades físico-químicas das séries homologas mais relevantes de $\mathrm{PBDE}^{64}$

\begin{tabular}{|c|c|c|c|}
\hline \multirow{2}{*}{ Propriedades } & \multicolumn{3}{|c|}{ PBDEs } \\
\hline & PentaBDE & OctaBDE & DecaBDE \\
\hline Formula molecular & $\mathrm{C}_{12} \mathrm{H}_{5} \mathrm{Br}_{5} \mathrm{O}$ & $\mathrm{C}_{12} \mathrm{H}_{2} \mathrm{Br}_{8} \mathrm{O}$ & $\mathrm{C}_{12} \mathrm{Br}_{10} \mathrm{O}$ \\
\hline Pressão de vapor & $2,2 \times 10^{-7}$ a $5,5 \times 10^{-7} \mathrm{~mm} \mathrm{Hg}$ a $25^{\circ} \mathrm{C}$ & $9,0 \times 10^{-10}$ a $1,7 \times 10^{-9} \mathrm{~mm} \mathrm{Hg}$ a $25^{\circ} \mathrm{C}$ & $3,2 \times 10^{-8} \mathrm{~mm} \mathrm{Hg}$ a $25^{\circ} \mathrm{C}$ \\
\hline Solubilidade em água & $13,3 \mu \mathrm{g} \mathrm{L} \mathrm{L}^{-1}$ a $25^{\circ} \mathrm{C}$ & $<1 \mu \mathrm{g} \mathrm{L}^{-1}$ a $25^{\circ} \mathrm{C}$ & $<1 \mu \mathrm{g} \mathrm{L}^{-1}$ a $25^{\circ} \mathrm{C}$ \\
\hline $\log \mathrm{K}_{\mathrm{OW}}$ & 6,64 a 6,97 & 6,29 & 6,26 \\
\hline Constante da Lei de Henry & $1,2 \times 10^{-5} \mathrm{~atm}-\mathrm{m}^{3} \mathrm{~mol}^{-1}$ & $7,5 \times 10^{-8} \mathrm{~atm}-\mathrm{m}^{3} \mathrm{~mol}^{-1}$ & $1,62 \times 10^{-6} \mathrm{~atm}-\mathrm{m}^{3} \mathrm{~mol}^{-1}$ \\
\hline
\end{tabular}


na ordem de centenas de $\mathrm{ng} \mathrm{g}^{-1}$. Em peixes, por exemplo, os níveis encontrados variaram de 1,5 a $100 \mathrm{ng} \mathrm{g}^{-1}$ (peso lipídico), na região dos Grandes Lagos (América do Norte), e de 30,6 a $281 \mathrm{ng} \mathrm{g}^{-1}$ (peso lipídico) em estuários de Taiwan. ${ }^{67,68} \mathrm{Na}$ atmosfera do leste da China, os níveis de PBDEs encontram-se em torno de $200 \mathrm{pg} \mathrm{m}^{-3} \mathrm{e}$, no sedimento de lagos da mesma região e áreas adjacentes, os níveis variaram de 0,02 a 46,6 ng g $^{-1}$ de peso seco. ${ }^{69,70} \mathrm{Já}_{\text {em regiões menos industria- }}$ lizados, as concentrações de PBDEs são menores; por exemplo, na Baía de Concepción valores para sedimentos ficaram próximos de $1 \mathrm{ng} \mathrm{g}^{-1}$ de peso seco e no Sul do Brasil o valor médio em bivalves foi de 6,83 $\mathrm{ng} \mathrm{g}^{-1}$ de peso lipídico. ${ }^{71,72}$ Entretanto, apesar dos resultados serem apresentados em termos de somatório de PBDEs, uma análise comparativa dos resultados deve levar em consideração o número de congêneres analisados em cada estudo, visto que isso pode influenciar significativamente na concentração reportada.

Além da alta industrialização, algumas regiões, como a China e países do sudeste da Ásia, também enfrentam problemas com a grande quantidade de lixo eletrônico, os chamados $e$-wastes, que contribuem para elevar os níveis ambientais de diversos contaminantes, dentre eles os PBDEs. ${ }^{73}$

Esses compostos são sabidamente tóxicos aos organismos, tendo como prováveis consequências a desregulação endócrina, carcinogenia e deficiência neurológica. Devido às semelhanças estruturais entre alguns PBDEs e os hormônios receptores da tireóide pode haver uma possível competição entre eles, causando alterações nas funções da tireóide, o que pode levar a mudanças das taxas metabólicas, síntese de proteína e desenvolvimento celular. ${ }^{74}$ A formação de tumores e câncer foi também associada à exposição a PBDEs. ${ }^{74}$ Estudo feito com homens e mulheres, com 60 anos em média, relacionou a dificuldade na fala e de memória com altos níveis de PCBs e PBDEs no sangue. ${ }^{75}$

\section{TETRABROMOBISFENOL A (TBBP-A)}

O tetrabromobisfenol A (TBBP-A) (Figura 3) é produzido pela bromação do bisfenol A (BPA) e é amplamente usado como retardante de chama em polímeros, tais como acrilonitrila-butadieno-estireno (ABS), resinas, adesivos, equipamentos elétricos e eletrônicos, eletrodomésticos, móveis e material de construção. ${ }^{76,77}$ Sua principal aplicação é em resina epóxi utilizada para produção de placas de circuito impresso, nas quais o teor de bromo pode chegar a $20 \%$ do peso. ${ }^{78} \mathrm{O}$ TBBP-A também pode ser usado como composto base para a produção de outros retardantes de chama comerciais, tais como tetrabromobisfenol A bis (2-hidroxietil éter), Tetrabromobisfenol A dibromopropileter, Tetrabromobisfenol A bis (alquil éter), Tetrabromobisfenol A oligômero de carbonato. ${ }^{76}$ Em função das suas propriedades físico-químicas (Tabela 3), o TBBP-A é considerado um composto lipofílico com grande afinidade por partículas, alta volatilidade e baixa solubilidade em água.

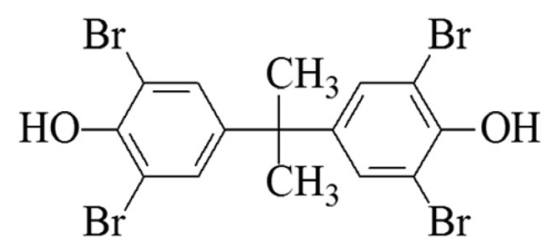

Figura 3. Estrutura química do Tetrabromobisfenol A (TBBP-A)

O TBBP-A é o retardante de chama bromado com o maior volume de produção global, ${ }^{6}$ estimada, em 2006, em 150 mil toneladas/ano. ${ }^{76}$ É produzido principalmente em Israel, Estados Unidos, Jordânia, Japão e China. ${ }^{6} \mathrm{O}$ uso de TBBP-A é permitido em todo o mundo e não há restrições sobre a produção deste e seus derivados. ${ }^{78} \mathrm{Com}$
Tabela 3. Propriedades físico-químicas do Tetrabromobisfenol A (TBBP-A) ${ }^{76}$

\begin{tabular}{lc}
\hline Propriedades & Value \\
\hline Fórmula molecular & $\mathrm{C}_{15} \mathrm{H}_{12} \mathrm{Br}_{4} \mathrm{O}_{2}$ \\
Pressão de vapor & $6,24.10^{-6} \mathrm{~Pa} \mathrm{a} 25^{\circ} \mathrm{C}$ \\
Solubilidade em água & $0,24 \mathrm{mg} \mathrm{L}$ a $25^{\circ} \mathrm{C}$ \\
Log $\mathrm{K}_{\text {Ow }}$ & 5,90 \\
Constante da Lei de Henry & $<9,87 \times 10^{-7} \mathrm{~atm}^{-\mathrm{m}^{3} \mathrm{~mol}^{-1}}$ \\
\hline
\end{tabular}

a proibição dos principais PBDEs nos EUA e Canadá, o TBBP-A tornou-se o retardante de chama bromado mais utilizado nestes países. ${ }^{79}$ Representou cerca de $60 \%$ do mercado global de BFRs em 2001, dos quais $70 \%$ foram usados como retardante de chama reativo em equipamentos elétricos e eletrônicos, $20 \%$ como aditivo em materiais poliméricos e $10 \%$ para a produção de derivados..$^{76,80} \mathrm{~A}$ Ásia é o maior consumidor de TBBP-A, visto que a maioria dos itens eletrônicos que utilizam placas de circuito eletrônicos são produzidos nesta área, especialmente na China. ${ }^{81}$

O TBBP-A pode ser aplicado tanto na forma reativa, ligando-se covalentemente com o grupo fenílico hidroxilado, fazendo parte da estrutura do polímero, como na forma aditiva, sendo simplesmente aderido/ misturado a este. ${ }^{82} \mathrm{~A}$ forma reativa representa a maior aplicação deste retardante de chama na indústria/mercado (90\%). Apesar de ser mais difícil, mesmo a forma reativa pode liberar TBBP-A ao ambiente. ${ }^{6,83,84}$ A exposição pode ocorrer através do vapor ou pó que são exalados de equipamentos (como televisores ou computadores) quando aquecidos durante o uso. ${ }^{6}$ Quando usado como aditivo nos polímeros, por não fazer parte da estrutura dos mesmos, pode ser facilmente liberado para o ambiente. Sendo assim, o TBBP-A pode ser liberado para o ambiente durante a produção, utilização e, principalmente, através da lixiviação de produtos que contenham na sua composição este retardante de chama. ${ }^{85}$ Incêndios acidentais ou incineração de materiais contendo TBBP-A causam preocupação, pois estes processos podem também resultar na geração de dioxinas e furanos bromados. ${ }^{86}$

O TBBP-A foi reportado em diferentes compartimentos ambientais: ar, solos e sedimentos. ${ }^{84} \mathrm{~A}$ revisão feita por Covaci et al. aponta para níveis significativos de TBBP-A em matrizes da biota marinha, incluindo invertebrados, peixes, aves e mamíferos, sendo as maiores concentrações encontradas em organismos do topo da cadeia alimentar. ${ }^{77}$

No ambiente o TBBP-A pode sofrer processos de volatilização, foto-oxidação química, oxidação, bioacumulação, e adsorção em partículas de sedimentos. ${ }^{87}$ Durante o processo de degradação pode ser transformado em derivados, tais como tri-, di-, mono-bromobisfenol A e bisfenol-A (BPA). ${ }^{56,88-90}$

Os caminhos biológicos pelos quais TBBP-A é degradado no ambiente são pouco conhecidos, porém, recentemente, foi demonstrado que um dos principais processos para a remoção do TBBP-A pode ser feita através da degradação microbiana. Em ambientes anaeróbicos o TBBP-A pode ser transformado em Bisfenol A (BPA) e em ambientes aeróbios para éter dimetílico de TBBP-A (TBBPA DME). ${ }^{87,91}$

A toxicidade do TBBP-A tem sido amplamente investigada em animais e plantas e, apesar de ter sido relatado como composto de baixa toxicidade, são relatados efeitos imunotóxico e neurotóxico em animais. ${ }^{56,92-94} \mathrm{Em}$ peixes observou-se diminuição da frequência cardíaca, edema e má formação da cauda, além de efeitos como desorientação, sonolência, diminuição da produção de ovos e do sucesso reprodutivo. ${ }^{91,95}$ Em ratos, o TBBP-A mostrou efeitos neurocomportamental, endócrino e reprodutivo. ${ }^{96-98}$ Também pode ser considerado como potencial desregulador endócrino, devido a sua semelhança estrutural com o hormônio da tireóide tiroxina (T4). 
Apesar dos efeitos apontados em laboratório, em razão das baixas concentrações encontradas e consequente reduzida exposição à biota, acredita-se que o TBBP-A não apresente risco ao ambiente e ao homem. ${ }^{6,99}$ No entanto, áreas com maior consumo de TBBP-A, a exemplo de algumas regiões da Ásia, onde foi observado um aumento na concentração desse contaminante no sedimento, requerem maior atenção devido aos efeitos deletérios associados à presença desse composto. ${ }^{100}$

Apesar da produção e do uso do TBBP-A não serem proibidos, diversos órgãos têm desenvolvido programas de monitoramento e de avaliações de impactos ambientais e na saúde humana. Por exemplo, a União Europeia incluiu o TBBP-A no REACH (Registration, Evaluation, Authorisation and Restriction of Chemicals) e a EPA tem coordenado diversos estudos de ocorrência e efeitos tóxicos. Já na Ásia, onde se localizam os maiores consumidores de TBBP-A, como China, Japão, Coreia e Taiwan, parece não haver iniciativa de órgãos ambientais para que a produção e o uso deste contaminante sejam proibidos. ${ }^{6}$ No Brasil não existem registros sobre seu uso, produção, exportação ou importação e nem mesmo informações sobre medidas reguladoras. ${ }^{23}$

\section{HEXABROMOCICLODODECANO (HBCD)}

O Hexabromociclododecano (HBCD) (Figura 4) é um retardante de chama do tipo aditivo que tem sido amplamente usado pelas indústrias de polímeros e têxteis nas últimas décadas. Seus principais usos são em espumas e resinas de poliestireno, posteriormente usadas pela indústria em móveis estofados, interiores de veículos, blocos usados na construção de residências, telhados, embalagens e equipamentos elétricos..$^{56}$

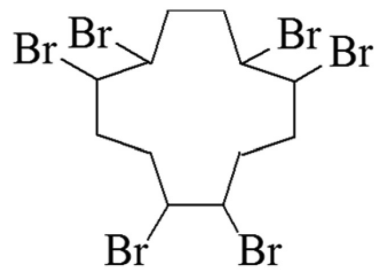

Figura 4. Estrutura química do Hexabromociclododecano (HBCD)

O HBCD é produzido a partir da bromação dos quatro isômeros do 1,5,9 ciclododecatrieno (CDTs) (trans, trans, trans-, trans, trans, cis-, trans, cis, cis-, e cis,cis,cis-CDT). Dependendo da constituição isomérica da mistura técnica de CDTs usada e das condições do processo industrial, misturas de HBCDs com diferentes composições e proporções entre isômeros são produzidas. De uma forma geral, 16 estereoisômeros de HBCDs são possíveis: seis diastoisômeros com seus pares de enantiômeros e quatro meso formas. ${ }^{101}$ Entre esses os mais comuns são os diasteroisômeros $\alpha-, \beta-, \gamma-\mathrm{HBCD}$ nas formas de enântionmero (+) e enantiômero (-). ${ }^{102} \mathrm{Na}$ mistura técnica esses isômeros estão presentes nas seguintes proporções: $1-12 \%$ para o $\alpha$-HBCD, $10-13 \%$ para o
$\beta$-HBCD e 75 - 89\% para o $\gamma$-HBCD (Tabela 4). ${ }^{101,103}$

Por ser um retardante de chama do tipo aditivo, ou seja, é apenas adicionado aos polímeros e não está quimicamente ligado, o HBCD é facilmente liberado dos produtos nos quais é usado e acumula nos diversos compartimentos ambientais. O HBCD foi detectado pela primeira vez em meados da década de 1990 em amostras de ar, esgoto e sedimento na Suécia e, desde então, tem sido detectado em vários compartimentos ambientais, biota e seres humanos. ${ }^{104-111}$ Além disso, estudos reportam sua ocorrência tanto em áreas urbanas e industrializadas, como em locais remotos..$^{73,105,107,112,113}$ Dessa forma, o HBCD é considerado um contaminante com distribuição ubíqua. Por se tratar de um composto orgânico não-volátil, estudos inferem que são transportados globalmente pelas correntes atmosféricas e oceânicas, adsorvidos as partículas. ${ }^{73}$

Embora alguns trabalhos reportem a ocorrência do diasteroisômero $\delta$-HCBD, os mais encontrados em matrizes ambientais são os $\alpha-, \beta$-, e $\gamma$-HBCD. ${ }^{107-108,110} \mathrm{O}$ padrão de distribuição entre eles é similar ao perfil das formulações comerciais, com predomínio do isômero $\gamma \cdot{ }^{101,105}$ Entretanto, em diversos trabalhos, principalmente na biota, a contribuição do $\alpha$-HBCD tem aumentado. ${ }^{109,114,115} \mathrm{Um}$ estudo realizado com peixes expostos a HBCD relatou que o $\alpha$-HBCD possui maiores valores de meia vida e de fator de bioacumulação que os demais isômeros, fator que explicaria a maior abundância de $\alpha$-HBCD em organismos. ${ }^{116}$

Poucos estudos avaliaram a toxicidade do $\mathrm{HBCD}$, porém há indícios de que apresenta uma elevada toxicidade, sendo capaz de induzir câncer, afetar o funcionamento do sistema hormonal e hepático. ${ }^{117}$ Também existem indícios de efeitos sobre a fertilidade e neurotoxicidade em mamíferos e de desregulação hormonal da tireóide. ${ }^{117} \mathrm{~A}$ exposição de filhotes de ratos a $\alpha$-HBCD mostrou um prejuízo no desenvolvimento do cérebro promovido pelo hormônio da tiroide (T3) ${ }^{117}$ Outro estudo observou alterações no comportamento reprodutivo de falcões, com diminuição no cuidado parental, na temperatura de incubação dos ovos durante a nidificação e na taxa de cortejo. ${ }^{118}$ Os diferentes isômeros também apresentam diferentes toxicidades. Du et $a l .{ }^{119}$ reportou inibição do crescimento, má-formação, atraso na eclosão dos ovos e decréscimo na taxa de sobrevivência de peixes relacionado, tanto com a concentração de exposição, quanto com o isômero ao qual os organismos foram expostos. Segundo os resultados desse mesmo estudo, o efeito dos isômeros sobre o crescimento dos organismos se daria na seguinte ordem: $\gamma$-HBCD $>\beta-H B C D>\alpha-H B C D$.

Segundo a Comissão Nacional de Segurança Química (CONASQ) e a Associação Brasileira da Indústria Química (Abiquim), o HBCD é usado no Brasil nos setores automotivos, de construção civil, eletrônica e têxtil. Sua concentração pode variar de 0,3 a $0,7 \%$, na forma de polistireno expansível, poliestireno extrudado e poliestireno de alto impacto para uso como isolamento térmico na indústria de construção civil e como aditivo retardante de chama para uso industrial, obedecendo a norma brasileira NBR $11948 .{ }^{22}$ No entanto, como o HBCD não possui código de identificação especifico (NCM), não é possível identificar as quantidades importadas e/ou exportadas. ${ }^{23}$

Tabela 4. Propriedades físico-químicas dos congêneres do $\mathrm{HBCD}^{120}$

\begin{tabular}{|c|c|c|c|c|}
\hline \multirow{2}{*}{ Propriedades } & \multicolumn{4}{|c|}{ HBCD } \\
\hline & $\alpha-\mathrm{HBCD}$ & $\beta-\mathrm{HBCD}$ & $\gamma$-HBCD & HBCD* \\
\hline Formula molecular & $\mathrm{C}_{12} \mathrm{H}_{18} \mathrm{Br}_{6}$ & $\mathrm{C}_{12} \mathrm{H}_{18} \mathrm{Br}_{6}$ & $\mathrm{C}_{12} \mathrm{H}_{18} \mathrm{Br}_{6}$ & $\mathrm{C}_{12} \mathrm{H}_{18} \mathrm{Br}_{6}$ \\
\hline Pressão de vapor & - & - & - & $6,3 \times 10^{-5} \mathrm{~Pa}$ a $21^{\circ} \mathrm{C}$ \\
\hline Solubilidade em água & $48,8 \pm 1,9 \mu \mathrm{g} \mathrm{L} \mathrm{L}^{-1}$ & $14,7 \pm 0,5 \mu \mathrm{g} \mathrm{L}^{-1}$ & $2,1 \pm 0,2 \mu \mathrm{g} \mathrm{L}^{-1}$ & $65,6 \mu \mathrm{g} \mathrm{L}^{-1}$ \\
\hline $\log \mathrm{K}_{\mathrm{OW}}$ & $5,07 \pm 0,09$ & $5,12 \pm 0,09$ & $5,47 \pm 0,10$ & 5,63 \\
\hline Constante da Lei de Henry & - & - & - & $7,40 \times 10^{-6}$ atm- $\mathrm{m}^{3} \mathrm{~mol}^{-1}$ \\
\hline
\end{tabular}

* mistura técnica 
Devido as suas características de bioacumulação, bioamagnificação, persistência e potencial para ser transportado a longas distâncias, o HBCD foi incluído, em 2013, no Anexo A da Convenção de Estocolmo. Entretanto, são aceitas exceções específicas para o seu uso em poliestireno expandido e poliestireno extrudido. O Brasil não possui legislação específica para o uso de $\mathrm{HBCD}$, mas como signatário da Convenção de Estocolmo deve atender às decisões associadas a inclusão no Anexo A, que envolve a eliminação do HBCD.

\section{OUTROS RETARDANTES DE CHAMA BROMADOS}

Com as restrições e/ou proibições no uso, produção e comercialização de alguns retardantes de chamas bromados, outros compostos bromados começaram a ganhar espaço como retardantes de chama. Uma relação dos principais produtos atualmente disponíveis, juntamente com algumas informações de uso e produção, encontra-se na Tabela 5.

Os dados de ocorrência desses compostos em matrizes ambientais

Tabela 5. Relação dos principais compostos bromados disponiveis, seus usos, nomes técnicos e empresas responsáveis pela sua produção

\begin{tabular}{|c|c|c|c|c|}
\hline Composto & Sigla & Uso & Companhia (Nome Técnico) & Referências \\
\hline Decabromodifenil Etano & DBDPE & Substituto para o DecaBDE. & $\begin{array}{c}\text { Albermale Corp. (Saytex } ® ~ 8010) \\
\text { (Chemtura Corp. (Firemaster } ® 2100)\end{array}$ & 121 \\
\hline $\begin{array}{l}\text { 1,2-bis(2,4,6 tribromofenoxi) } \\
\text { etano }\end{array}$ & ВТВРЕ & Substituto do OctaBDE. & Chemtura Corp. (FF-680) & 121 \\
\hline $\begin{array}{l}\text { Bis(2-etilhexil) } \\
\text { tetrabromoftalato }\end{array}$ & ТВРН ou ВЕНТВР & Substituto do PentaBDE. & $\begin{array}{l}\text { Chemtura Corp. (Firemaste } 550= \\
\text { TBPH + TBB1, BZ }-54, \mathrm{DP}-45 \text { ) }\end{array}$ & 121 \\
\hline Hexabromobenzeno & $\mathrm{HBBz}$ & $\begin{array}{l}\text { Aditivo em papel, madeira, } \\
\text { têxteis, eletro-eletrônicos. }\end{array}$ & Shou Guang Longfa Chemical Co. Ltd. & 121 \\
\hline Pentabromotolueno & PBT & $\begin{array}{l}\text { Polietileno, polipropileno, } \\
\text { poliestireno, látex de SBR, } \\
\text { borrachas. }\end{array}$ & $\begin{array}{c}\text { ICL Industrial Corporation (FR-105) } \\
\text { Albemarle Corp. } \\
\text { Berk Ltd. (Flammex 5-BT) }\end{array}$ & 121 \\
\hline Pentabromoetilbenzeno & PBEB & $\begin{array}{l}\text { Resinas de poliéster termofixas } \\
\text { (plascas de circuitos, têxteis, } \\
\text { adesivos, cabos e fiações, } \\
\text { espumas de poliuretano). }\end{array}$ & $\begin{array}{c}\text { Albemarle Corp. } \\
\text { ICL Industrial Corporation (FR-105) } \\
\text { Chemtura Corp } \\
\text { Shou Guang Longfa Chemical Co. Ltd. }\end{array}$ & 121 \\
\hline $\begin{array}{l}\text { Tetrabromobisfenol A dialil } \\
\text { éter }\end{array}$ & TBBPA-DAE & Espumas de poliestireno. & Chemtura Corp. (BE-51) & 121 \\
\hline $\begin{array}{l}\text { Tetrabromobisfenol A-bis(2,3- } \\
\text { dibromopropil éter) }\end{array}$ & TBBPA-DBPE & $\begin{array}{c}\text { Usado em produtos plásticos } \\
\text { como encanamentos, } \\
\text { barricadas, exaustores e } \\
\text { eletrônicos. }\end{array}$ & $\begin{array}{c}\text { ICL Industrial Corporation (FR-720) } \\
\text { Albemarle Corp. } \\
\text { Chemtura Corp. (PE-68) } \\
\text { JiangSu HaoHua Fine Chemical Co. Ltd. } \\
\text { Weidong Inter. Group Ltd. }\end{array}$ & 121 \\
\hline $\begin{array}{l}\text { Tetrabromobisfenol A } \\
\text { dihidroxietil eter }\end{array}$ & TBBPA-DHEE & $\begin{array}{l}\text { Usado em polímeros e } \\
\text { revestimentos. }\end{array}$ & $\begin{array}{l}\text { Chemtura Corp. (BA-50P e BA-50) } \\
\text { Teijin Chemicals Ltd. (Fireguard 3600) }\end{array}$ & 121 \\
\hline $2,4,6$ - tribromofenol & TBP & $\begin{array}{l}\text { BFR aditivo, agente antifungo } \\
\text { e intermediário químico. }\end{array}$ & $\begin{array}{c}\text { Chemtura Corp. (PH-73FF) } \\
\text { ICL Industrial Corporation (FR-613) } \\
\text { Weidong Int. Grup, Ltd. } \\
\text { Pode ser produzido por algas marinhas } \\
\text { Produto da metabolização de PBDEs, OH- } \\
\text { PBDEs e metoxi-PBDEs. }\end{array}$ & 121 \\
\hline $2,4,6$ - tribromofenil alil éter & ATE & $\begin{array}{l}\text { Espuma de poliestireno, } \\
\text { polietireno expandível. }\end{array}$ & Chemtura Corp. (PHE-65) & 121 \\
\hline $\begin{array}{l}\text { 1,2-dibromo-4-(1,2- } \\
\text { dibromoetil)ciclohexano }\end{array}$ & TBECH & $\begin{array}{l}\text { Material para isolametno de } \\
\text { casas, adesivos, tecidos, cabos } \\
\text { elétricos, plásticos duros, } \\
\text { materiais de construção. }\end{array}$ & Albemarle Corp.(Saytex BCL-462) & 121 \\
\hline $\begin{array}{l}\text { Hexaclorociclopentadienil- } \\
\text { dibromociclooctano }\end{array}$ & $\mathrm{HCDBCO}$ & Polímero estirenico. & Não há informações disponíveis. & 78 \\
\hline $\begin{array}{l}\text { Tris }(2,3 \text { - dibromopropil }) \\
\text { isocianureto }\end{array}$ & TBC & $\begin{array}{c}\text { Poliuretano, PVC, ABS, } \\
\text { poliéster insaturados, borracha } \\
\text { sintética, fibras. }\end{array}$ & Diversas companhias chinesas. & 78 \\
\hline $\begin{array}{l}\text { Octabromo-1,3,3-trimetil } \\
\text {-1-1fenilindano }\end{array}$ & OBIND & $\begin{array}{c}\text { Não há informações } \\
\text { disponíveis. }\end{array}$ & ICL Industrial Corporation (FR-1808) & 78 \\
\hline Anidro Tetrabromoftálico & TBPhA ou TPA & $\begin{array}{l}\text { Poliéster insaturados, espimas } \\
\text { de poliuretanso rígidas, papel, } \\
\text { têxteis, lã, resinas epóxi. }\end{array}$ & $\begin{array}{l}\text { Chemtura Corp. (PHT4) } \\
\text { Albemarle Corp.(Saytex RB-49) }\end{array}$ & 121 \\
\hline Acrilato de Pentabromobenzil & PBBA & $\begin{array}{l}\text { BFR intermediário aplicado } \\
\text { em polibutilenotereftalato, } \\
\text { polietilenoteraftalato e } \\
\text { polímeros ABS }\end{array}$ & Não há informações disponíveis. & 78 \\
\hline $\begin{array}{l}1,2,5,6 \text { - tetrabromociclo- } \\
\text { octano }\end{array}$ & $\mathrm{TBCO}$ & $\begin{array}{c}\text { Não há informações } \\
\text { disponíveis. }\end{array}$ & Albemarle Corp.(Saytex BCL-48) & 78 \\
\hline
\end{tabular}

${ }^{1} \mathrm{TBB}=$ 2-etilhexil-2,3,4,5-tetrabromobenzoato. 
são, em geral, informações colaterais em estudos sobre PBDEs, PBBs, HBCDs e TBBP-A. ${ }^{39}$ No Brasil, apenas um estudo foi encontrado em que três compostos (Decabromodifeniletano (DBDPE), Hexabromobenzeno (HxBB) e Pentabromoetilbenzeno (PBEB)) foram analisados em amostras de fígado de Pontoporia blainville da costa sul e sudeste do Brasil. ${ }^{39}$

\section{BFRs NO BRASIL: OCORRÊNCIA E TENDÊNCIAS}

Poucos são os trabalhos que avaliaram a ocorrência de BFRs em amostras ambientais no Brasil. Um compilado do que se encontra publicado é apresentado na Tabela 6 . Como pode ser observado, quase a totalidade dos estudos é relacionada à ocorrência de PBDEs em mamíferos marinhos, com poucos estudos realizados em amostras de peixes, sedimento, tecido adiposo humano e bivalves..$^{39,72,122-128}$ Além disso, os poucos estudos realizados foram com amostras oriundas apenas das regiões sul e sudeste do Brasil. A maioria dos níveis de PBDEs detectados em amostras do Brasil é baixa. No entanto, alguns estudos reportaram valores similares aos encontrados em animais de regiões sabidamente contaminadas do Hemisfério Norte, como por exemplo os valores encontrados em golfinhos do litoral do Rio de Janeiro e São Paulo. ${ }^{129,130}$

\section{RETARDANTES DE CHAMAS NÃO HALOGENADOS (ALTERNATIVAS)}

Com a crescente preocupação com os efeitos deletérios dos retardantes de chamas bromados e seu gradual banimento, surgiu

Tabela 6. Ocorrência de BFRs em amostras ambientais no Brasil

\begin{tabular}{|c|c|c|c|c|c|c|c|}
\hline Tipo de Amostra & Local & Ano & $\mathrm{n}$ & $\sum$ PBDEs $\left(\mathrm{ng} \mathrm{g}^{-1}\right)$ & $\sum$ HBCDs & $\sum$ PBBs & Referências \\
\hline \multicolumn{8}{|c|}{ Bivalves } \\
\hline Perna Perna & RS & 2009 & & $<\mathrm{LDM}-16^{\mathrm{a}}$ & - & - & 131 \\
\hline \multicolumn{8}{|c|}{ Aves } \\
\hline Spheniscus magellanicus (fígado) & SP, SC e RS & $2008-2012$ & 102 & $1,18-9,69^{\mathrm{b}}$ & - & - & 132 \\
\hline \multicolumn{8}{|c|}{ Crustáceos } \\
\hline Hepatus pudibundus & SP & 2002 & & $0,37-1,44^{a}$ & - & - & 133 \\
\hline Callinectes danae & SP & 2002 & & $1,23-4,74^{\mathrm{a}}$ & - & - & 133 \\
\hline \multicolumn{8}{|c|}{ Peixes } \\
\hline Katsuwonus pelamis (músculo/fígado) & Costa do Brasil & 2000 & 5 & $13^{\mathrm{b}}$ & $0,28^{b}$ & - & 110,134 \\
\hline Lepidopus caudatus (fígado) & $\mathrm{RJ}$ & - & 10 & Média $=10.2^{\mathrm{a}}$ & - & - & 124 \\
\hline \multirow[t]{2}{*}{ Micropogonias furnieri (fígado) } & RJ & - & 10 & Média $=3.45^{\mathrm{a}}$ & - & - & 124 \\
\hline & RS & 2012 & 60 & $<\mathrm{LDM}$ & - & - & 135 \\
\hline Megachasma pelagios (fígado e músculo) & $\mathrm{RJ}$ & 2009 & 1 & $<\mathrm{LDM}$ & - & - & 136 \\
\hline \multicolumn{8}{|c|}{ Mamíferos Marinhos } \\
\hline Sotalia guianensis (fígado) & RJ & & & $13-1620^{b}$ & - & - & 129 \\
\hline Stenella frontalis (fígado) & RJ & $1994-2006$ & & $96-2440^{b}$ & - & - & 129 \\
\hline Pseudorca crassidens (fígado) & RJ & $1994-2006$ & & $1210-5960^{b}$ & - & - & 129 \\
\hline Tursiops truncatus (fígado) & RJ & $1994-2006$ & & $270-1350^{b}$ & - & - & 129 \\
\hline Steno bradanensis (fígado) & RJ & $1994-2006$ & & $360-1600^{b}$ & - & - & 129 \\
\hline Delphinus delphis (fígado) & RJ & $1994-2006$ & & $125-240^{\mathrm{b}}$ & - & - & 129 \\
\hline Stenella attenuate (fígado) & RJ & $1994-2006$ & 1 & $1215^{\mathrm{b}}$ & - & - & 129 \\
\hline Stenella longirostris (fígado) & RJ & $1994-2006$ & 1 & $150^{\mathrm{b}}$ & - & - & 129 \\
\hline Stenella coeruleoalba (fígado) & RJ & $1994-2006$ & 1 & $210^{b}$ & - & - & 129 \\
\hline Lagenodelphis hosei (fígado) & $\mathrm{RJ}$ & $1994-2006$ & & $3-28^{b}$ & - & - & 129 \\
\hline$\underline{\text { Stenella frontalis (gordura) }}$ & SP & $2004-2007$ & 9 & $23-1327^{b}$ & - & - & 130 \\
\hline \multirow{9}{*}{ Pontoporia blainvillei (gordura e fígado) } & ES & $1994-2009$ & 13 & $9,18-578^{b}$ & - & $<0,15-57,5$ & 39 \\
\hline & RJ & $1994-2009$ & 1 & $37,4^{\mathrm{b}}$ & - & 46 & 39 \\
\hline & SP & $1994-2009$ & 12 & $12,6-613^{b}$ & - & $<0,15-44,9$ & 39 \\
\hline & PR & $1994-2009$ & 3 & $7,92-157^{b}$ & - & $<0,15-15,4$ & 39 \\
\hline & $\mathrm{SC}$ & $1994-2009$ & 11 & $31,5-1797^{b}$ & - & $<0,15-17,25$ & 39 \\
\hline & RS & $1994-2009$ & 13 & $14,2-60,3^{b}$ & - & $<0,15-9,12$ & 39 \\
\hline & RS & 1994-2004 & & $13,4-65^{b}$ & - & - & 122 \\
\hline & SP & & & $93,6-655,9^{\mathrm{b}}$ & - & - & 122 \\
\hline & RJ & 2011-2012 & 9 & $13,84-36,94^{\mathrm{b}}$ & - & - & 137 \\
\hline Sotalia guianensis (fígado) & $\mathrm{RJ}$ & - & 10 & Média $=53^{\mathrm{a}}$ & - & - & 124 \\
\hline \multicolumn{8}{|c|}{ Sedimento } \\
\hline- & PR & $2011-2015$ & 32 & $<\operatorname{LDM}(<0,05)^{\mathrm{c}}$ & - & - & 127 \\
\hline \multicolumn{8}{|c|}{$\operatorname{Ar}\left(\mathrm{pg} \mathrm{m}^{-3}\right)$} \\
\hline - & $\begin{array}{c}\text { Atlântico Sul } \\
\text { (próximo a. Costa) }\end{array}$ & 2016 & 5 & $0,69-2,58$ & - & - & 138 \\
\hline- & Distribuição global & 2005 & & - & $<0,1-190$ & - & 139 \\
\hline
\end{tabular}

${ }^{\mathrm{a}}$ peso úmido, ${ }^{\mathrm{b}}$ peso lipídico e ${ }^{\mathrm{c}}$ peso seco. 
a necessidade de substituição desses compostos. Uma das alternativas para a substituição dos BFRs são os chamados Retardantes de Chamas Não Halogenados (HFFRs), dos quais pode-se destacar os retardantes de chama inorgânicos, compostos organofosforados e seus sais, retardantes de chama orgânicos à base de nitrogênio e sistemas intumescentes. ${ }^{140}$

Esses retardantes já são encontrados no mercado, porém pouco se sabe sobre seu comportamento ambiental e seus riscos à saúde humana. ${ }^{140}$ Um estudo recente demonstrou que retardantes de chama fosforados estão amplamente distribuídos pela atmosfera da América Latina, incluindo amostras do sul e nordeste do Brasil. ${ }^{141}$ Sendo assim, o uso de novos compostos não halogenados deve ser feito com cautela já que as poucas informações sobre a sua persistência, bioacumulação e toxicidade deixam dúvida se realmente seriam menos problemáticos do que os BFRs ao ambiente e seres humanos.

\section{CONSIDERAÇÕES FINAIS}

De maneira geral, os BFRs são compostos de grande potencial tóxico e que, apesar de já terem sido proibidos em alguns países, ainda são bastante usados principalmente por países em desenvolvimento. Além disso, novas fontes de BFRs, como o e-waste oriundo de atividades de reciclagem, tem gerado preocupação nas últimas décadas. Da mesma forma, compostos que estariam indisponíveis em geleiras ou na pergelissolo podem ser tornar disponíveis devido ao aquecimento associado às mudanças climáticas. ${ }^{142}$

Apesar do Brasil ser signatários da Convenção de Estocolmo, estando comprometido com a não comercialização de produtos contendo alguns dos BFRs ou seu uso direto, há pouca informação disponível sobre a produção e comercialização atual e histórica desses compostos no Brasil. Além disso, é sabido que muitos dos bens de consumo utilizados aqui, desde produtos eletrônicos até utensílios domésticos, são provenientes de importação. Muitos destes produtos são oriundos da China, por exemplo, país que sabidamente faz uso de BFRs e onde, até o momento, não há previsão do seu banimento. Fica evidente que não há um controle efetivo da entrada no mercado brasileiro dos bens de consumo que possam conter estes compostos, tão pouco uma legislação pertinente que vise a sua regulamentação. A ocorrência de BFRs em amostras ambientais da costa brasileira evidencia o uso desses produtos no Brasil..$^{39,110,122,124,129,131,134}$ Portanto, é fundamental que este tema seja abordado com maior propriedade, por meio de estudos que permitam uma avaliação detalhada do status da contaminação atual e futura, bem como pela implementação de legislações específicas no país.

\section{MATERIAL SUPLEMENTAR}

Os números CAS dos compostos citados neste trabalho estão compilados na Tabela 1S, disponível em http://quimicanova.sbq.org. br, na forma de arquivo PDF, com acesso livre.

\section{AGRADECIMENTOS}

Esse trabalho teve apoio da CAPES (bolsa de mestrado: Michelle C. Pieroni e PNPD no. PNPD2509091: Juliana Leonel) e do CNPq (PQ 312341/2013-0 - Gilberto Fillmann). Os autores também agradecem à Gabriela M. Ignácio pela confecção das figuras.

\section{REFERÊNCIAS}

1. Alaee, M.; Environ. Int. 2003, 29, 683.

2. http://www.inchem.org/documents/ehc/ehc/ehc192.htm, acessada em Setembro 2016.
3. http://www.flameretardants-online.com/web/en/106/7ae3d32234954e2 8e661e506e284da7f.htm , acessada em Setembro 2016

4. Freedonia; Addit. Polym. 2013, 10.

5. Troitzsch, J. H.; Chem. Today 1998, 16, 19.

6. www.bsef.com, acessada em Setembro 2016.

7. de Wit, C.; Herzke, D.; Vorkamp, K.; Sci. Total Environ. 2010, 408, 2885.

8. Leonel, J.; Tese de Doutorado, Universidade de São Paulo, Brasil, 2007.

9. Yogui, G. T.; Sericano, J. L.; Environ. Int. 2009, 35, 655.

10. Hatje, V.; Costa, M. F.; Cunha, L. C.; Quim. Nova 2013, 36, 1497.

11. Bila, D. M.; Dezotti, M.; Quim. Nova 2007, 30, 651.

12. Pestana, C. R.; Borges, K. B.; Fonseca, P.; Oliveira, D. P.; Rev. Bras. Toxicol. 2008, 21, 41.

13. McKinney, M. A.; Letcher, R. J.; Aars, J.; Born, E. W.; Branigan, M.; Dietz, R.; Evans, T. J.; Gabrielsen, G. W.; Peacock, E.; Sonne, C.; Environ. Int. 2011, 37, 365 .

14. Evenset, A.; Christensen, G. N.; Carroll, J.; Zaborska, A.; Berger, U.; Herzke, D.; Gregor, D.; Environ. Pollut. 2007, 146, 196.

15. Borghesi, N.; Corsolini, S.; Leonards, P.; Brandsma, S.; de Boer, J.; Focardi, S.; Chemosphere 2009, 77, 693.

16. http://chm.pops.int/Portals/0/download.aspx ?d=UNEP-POPS-COPCONVTEXT-2009.En.pdf, acessada em Setembro 2016.

17. http://www.pic.int/theconvention/chemicals/annexiiichemicals/ tabid/1132/langua ge/en-us/default.aspx, acessada em Setembro 2016.

18. http://www25.senado.leg.br/web/atividade/materias/-/materia/90884, acessada em Setembro 2016.

19. http://www.comprasnet.gov.br/legislacao/legislacaoDetalhe. asp?ctdCod=295, acessada em Setembro 2016.

20. Di Carlo, J.; Seifter, J.; Decarlo, V. J.; Environ. Health. Perspect. 1978 , 23,351 .

21. Hardy, M. L.; Chemosphere 2002, 46, 717.

22. http://www.mma.gov.br/images/arquivo/80037/Convencao $\% 20$ de $\% 20$ Estocolmo/Inventarios/livro_inventario_uso_industrial_novas\%20 correcoes.pdf, acessada em Setembro 2016.

23. Ministério do Meio Ambiente, comunicação pessoal, 2015.

24. http://aliceweb.mdic.gov.br/, acessada em Setembro 2016.

25. Terrell, M. L.; Berzen, A. K.; Small, C. M.; Cameron, L. L.; Wirth, J. J.; Marcus, M.; Environ. Health 2009, 8, 35.

26. Sundstrom, G.; Hutzinger, O.; Safe, S.; Zitko, V.; Canada, E.; Sci. Total Environ 1976, 6, 15.

27. http://chm.pops.int/TheConvention/POPsReviewCommittee/Reports/ tabid/2301/ctl/Download/mid/7538/Default.aspx id=77\&ObjID=4867, acessada em Setembro 2016.

28. Ibhazehiebo, K.; Iwasaki, T.; Xu, M.; Shimokawa, N.; Koibuchi, N.; Neurosci. Lett. 2011, 32, 1.

29. Pomerantz, I.; Burke, J.; Firestone, D.; Environ. Health. Perspect. 1978, $24,133$.

30. Henck, W. H.; Mattsson, J. L.; Rezabek, D. H.; Carlson, C. L.; Rech, R. H.; Neurotoxicol. Teratol. 1994, 16, 391.

31. Hesse, J. L.; Powers, R. A.; Environ. Health. Perspect. 1978, 23, 19.

32. http://www.atsdr.cdc.gov/toxprofiles/TP.asp?id=529\&tid=94, acessada em Setembro 2016.

33. Von der Recke, R.; Vetter, W.; Chemosphere 2008, 71, 352.

34. Gierón, J.; Grochowalski, A.; Chrza, R.; Chemosphere 2010, 78, 1272.

35. Fernandez, M. F.; Araque, P.; Kiviranta, H.; Molina-molina, J. M.; Neurotoxicology 2007, 66, 377.

36. Valciukas, J. A.; Lilis, R.; Wolff, M. S.; Anderson, H. A.; Environ. Health Perspect. 1978, 23, 199.

37. Chanda, J. J.; Anderson, H. A.; Glamb, R. W.; Lomatch, D. L.; Wolff, M. S.; Voorhees, J. J.; Selikoff, I. J.; Environ. Res. 1982, 29, 97.

38. Zhao, G.; Wang, Z.; Zhou, H.; Zhao, Q.; Sci. Total Environ. 2009, 407, 4831 . 
39. Alonso, M. B.; Eljarrat, E.; Gorga, M.; Secchi, E. R.; Bassoi, M.; Barbosa, L.; Bertozzi, C. P.; Marigo, J.; Cremer, M.; Domit, C.; Azevedo, A. F.; Dorneles, P. R.; Barceló, D.; Environ. Pollut. 2012, 170, 152.

40. Clarke, B.; Porter, N.; Symons, R.; Marriott, P.; Ades, P.; Stevenson, G.; Blackbeard, J.; Chemosphere 2008, 73, 980.

41. Montie, E. W.; Letcher, R. J.; Reddy, C. M.; Moore, M. J.; Rubinstein, B.; Hahn, M. E.; Mar. Pollut. Bull. 2010, 60, 1160.

42. Hu, G. C.; Luo, X. J.; Dai, J. Y.; Zhang, X. L.; Wu, H.; Zhang, C. L.; Guo, W.; Xu, M-Q.; Mai, B-X.; Wei, F. W.; Environ. Sci. Technol. 2008, 42,4704

43. Blanck, H. M.; Marcus, M.; Hertzberg, V.; Tolbert, P. E.; Rubin, C.; Henderson, A. K.; Zhang, R. H.; Environ. Health. Perspect. 2000, 108, 147.

44. Damstra, T.; Jurgelski, W.; Posner, H. S.; Vouk, V. B.; Bernheim, N. J.; Guthrie, J.; Luster, M.; Falk, H. L.; Environ. Health. Perspect. 1982, 44, 175.

45. Bursian, S. Em Veterinary Toxicology; Gupta, R. C., ed.; Elsevier, cap. 55.

46. Zhu, L.; Ma, B.; Li, J.; Wu, Y.; Gong, J.; Chemosphere 2009, 74, 1429.

47. Chen, Z. J.; Liu, H. Y.; Cheng, Z.; Man, Y. B.; Zhang, K. S.; Wei, W.; Du, J.; Wong M-H.; Wang, H. S.; Environ. Int. 2014, 73, 77.

48. Ecobichon, D. J.; Hidvegi, S.; Comeau, A. M.; Cameron, P. H.; Toxicology 1983, 28, 51.

49. Rozman, K. K.; Rozman, T. A.; Williams, J.; Greim, H. A.; J. Toxicol Environ. Health. 1982, 9, 611.

50. http://www.inchem.org/documents/ehc/ehc/ehc152.htm, acessada em Julho 2015.

51. Anderson, H. A. et al. Unanticipated Prevalence of Symptoms Among Dairy Farmers in Michigan and Wisconsin. Environ. Health Perspect. 1978, 23, 217.

52. Davis, S. I.; Blanck, H. M.; Hertzberg, V. S.; Tolbert, P. E.; Rubin, C.; Cameron, L. L.; Henderson, A. K.; Marcus, M.; Environ. Health 2005 , $4,15$.

53. Sweeney, A. M.; Symanski, E.; Environ. Res. 2007, 105, 370.

54. Neufeld, M. L.; Sittenfeld, M.; Wold, K. F.; USEPA 1977, 560/6-77-017.

55. http://chm.pops.int/Implementation/NIPs/Guidance/ GuidancefortheinventoryofPBDEs/tabid/3171/Default.aspx, acessada em Setembro 2016.

56. de Wit, C. A.; Chemosphere 2002, 46, 583.

57. Guerra, P.; Alaee, M.; Eljarrat, E.; Barceló, D. Em Brominated Flame Retardants; Eljarrat, E., Barceló, D., eds.; Springer: Berlin, 2011, cap.1.

58. http://www.inchem.org/documents/ehc/ehc/ehc162.htm, acessada em Setembro 2016

59. Rahman, F.; Langford, K. H.; Scrimshaw, M. D.; Lester, J. N.; Sci. Total Environ. 2001, 275, 1

60. Wong, A.; Lei, Y. D.; Alaee, M.; Wania, F.; J. Chem. Eng. Data 2001, 46, 239.

61. La Guardia, M. J.; Hale, R. C.; Harvey, E.; Environ. Sci. Technol. 2006, $40,6247$.

62. Park, J-S.; Holden, A.; Chu, V.; Newsome, S.D.; Henry, R.W.; Wang, M.; Kim, M.; Rhee, A.; Patel, P.; Fong, A.; Linthicum, J.; Hooper K.; Organohalogen Compd. 2009, 71, 820.

63. http://chm.pops.int/TheConvention/POPsReviewCommittee/ POPRCRecommendations/tabid/243/ctl/Download/mid/13251/Default. aspx id=53\&ObjID=16698, acessada em Setembro 2016.

64. http://www.atsdr.cdc.gov/ToxProfiles/tp.asp?id=901\&tid=183, acessada em Setembro 2016.

65. Shanmuganathan, D.; Megharaj, M.; Chen, Z.; Naidu, R.; Pollut. Bull. 2011, 63, 154 .

66. Benedict, R. T.; Stapleton, H. M.; Letcher, R. J.; Mitchelmore, C. L.; Chemosphere 2007, 69, 987.

67. Peng, J.; Huang, C.-W.; Weng, Y.-M.; Yak, H.-K.; Chemosphere 2007, 66, 1990.
68. Pérez-fuentetaja, A.; Lupton, S.; Clapsadl, M.; Samara, F.; Gatto, L.; Biniakewitz, R.; Aga, D. S.; Chemosphere 2010, 81, 541.

69. Qiu, X.; Zhu, T.; Hu, J.; Chemosphere 2010, 80, 1207.

70. Wu, F.; Guo, J.; Chang, H.; Liao, H.; Zhao, X.; Mai, B.; Xing, B.; Environ. Pollut. 2012, 162, 262.

71. Pozo, K.; Kuku ka, P.; Va ková, L.; P ibylová, P.; Klánová, J.; Rudolph, A.; Banguera, Y.; Monsalves, J.; Contreras, S.; Barra, R.; Ahumada, R.; Mar. Pollut. Bull. 2015, 95, 480.

72. Pieroni, M. C.; Monografia, Universidade Federal do Rio Grande, Brasil, 2007.

73. Law, R. J.; Covaci, A.; Harrad, S.; Herzke, D.; Abdallah, M. A.-E.; Fernie, K.; Toms, L-M. L.; Takigami, H.; Environ. Int. 2014, 65, 147.

74. Vonderheide, A. P.; Mueller, K. E.; Meija, J.; Welsh, G. L.; Sci. Total Environ. 2008, 400, 425.

75. Fitzgerald, E. F.; Shrestha, S.; Gomez, M. I.; Mccaffrey, R. J.; Zimmerman, E. A.; Kannan, K.; Hwang, S.; Neurotoxicology 2012, 33, 8.

76. http://echa.europa.eu/documents/10162/32b000fe-b4fe-4828-b3d3$93 \mathrm{c} 24 \mathrm{c} 1 \mathrm{cdd} 51$, acessada em Setembro 2016

77. Lévy-bimbot, M.; Major, G.; Courilleau, D.; Blondeau, J.; Lévi, Y.; Chemosphere 2012, 87, 782.

78. Covaci, A.; Voorspoels, S.; Abdallah, M. A. E.; Geens, T.; Harrad, S.; Law, R. J.; J. Chromatogr. A 2009, 1216, 346.

79. Bastos, P. M.; Eriksson, J.; Green, N.; Bergman, Å.; Chemosphere 2013, $70,1196$.

80. Law, R. J.; Allchin, C. R.; de Boer, J.; Covaci, A.; Herzke, D.; Lepom, P.; Morris, S.; Tronczynski, J.; de Wit, C. A.; Chemosphere 2006, 64, 187

81. He, M. J.; Luo, X. J.; Yu, L. H.; Wu, J. P.; Chen, S. J.; Mai, B. X.; Environ. Pollut. 2013, 179, 105.

82. h t t p : / / s u s tain ableproduction.org/downloads / AternativestoTBBPAandHBCD.pdf, acessada em Setembro 2016.

83. http://edz.bib.uni-mannheim.de/daten/edz-a/gdgv/11/flame_retardant_ substances_study_en.pdf, acessada em Setembro 2016.

84. Birnbaum, L. S.; Staskal, D. F.; Environ. Health Perspect. 2004, 112, 9.

85. Sjödin, A.; Patterson, D. G.; Environ. Int. 2003, 29, 829.

86. https://ec.europa.eu/jrc/en/publication/eur-scientific-and-technicalresearch-reports/european-union-risk-assessment-report-human-healthaddendum-april-2008-44, acessada em Setembro 2016.

87. Chang, B. V; Yuan, S. Y.; Ren, Y. L.; Chemosphere 2012, 87, 535.

88. Brenner, A.; Mukmenev, I.; Abeliovich, A.; Kushmaro, A.; Ecotoxicology 2006, 15, 399.

89. Eriksson, J.; Rahm, S.; Green, N.; Jakobsson, E.; Chemosphere 2004, $54,117$.

90. Gerecke, A. C.; Giger, W.; Hartmann, P. C.; Heeb, N. .; Kohler, H. P. E.; Schmid, P.; Zennegg, M.; Kohler, M.; Chemosphere 2006, 64, 311.

91. McCormick, J. M.; Paiva, M. S.; Haggblom, M. M.; Cooper, K. R.; White, L. A.; Aquat. Toxicol. 2010, 100, 255.

92. Debenest, T.; Gagné, F.; Petit, A.; André, C.; Kohli, M.; Blaise, C.; Comp. Biochem. Physiol., Part C: Toxicol. Pharmacol. 2010, 152, 407.

93. Sun, Y.; Guo, H.; Yu, H.; Wang, X.; Wu, J.; Xue, Y.; Chemosphere 2008, $70,1787$.

94. Pullen, S.; Boecker, R.; Tiegs, G.; Toxicology 2003, 184, 11.

95. Kuiper, R. V.; Van den Brandhof, E. J.; Leonards, P. E. G.; Van der Ven, L. T. M.; Wester, P. W.; Vos, J. G.; Arch. Toxicol. 2007, 81, 1.

96. Lilienthal, H.; Verwer, C. M.; Van der Ven, L. T. M.; Piersma, A. H.; Vos, J. G.; Toxicology 2008, 246, 45.

97. Nakajima, A.; Saigusa, D.; Tetsu, N.; Yamakuni, T.; Tomioka, Y.; Hishinuma, T.; Toxicol. Lett. 2009, 189, 78.

98. Van der Ven, L. T. M.; Van de Kuil, T.; Verhoef, A.; Verwer, C. M.; Lilienthal, H.; Leonards, P. E. G.; Piersma, A. H.; Toxicology 2008, 245, 76.

99. http://echa.europa.eu/documents/10162/32b000fe-b4fe-4828-b3d3$93 \mathrm{c} 24 \mathrm{c} 1 \mathrm{cdd} 51$, acessada em Setembro 2016 
100. Zhang, X.-L.; Luo, X.-J.; Chen, S.-J.; Wu, J.-P.; Mai, B.-X.; Environ. Pollut. 2009, 157, 1917.

101. Heeb, N. V; Schweizer, W. B.; Kohler, M.; Gerecke, A. C.; Chemosphere 2005, 61, 65 .

102. Koch, C.; Schmidt-Kötters, T.; Rupp, R.; Sures, B.; Environ. Pollut. 2015, 199, 26

103. Wu, T.; Wang, S.; Huang, H.; Zhang, S.; J. Agric. Food Chem. 2012, 60, 8528.

104. Sellström, U.; Bignert, A.; Kierkegaard, A.; Häggberg, L.; de Wit, C. A.; Olsson, M.; Jansson, B.; Environ. Sci. Technol. 2003, 37, 5496.

105. Feng, A. H.; Chen, S. J.; Chen, M. Y.; He, M. J.; Luo, X. J.; Mai, B. X.; Mar. Pollut. Bull. 2012, 64, 919.

106. Haukås, M.; Hylland, K.; Nygård, T.; Berge, J. A.; Mariussen, E.; Sci. Total Environ. 2010, 408, 5910.

107. Ichihara, M.; Yamamoto, A.; Takakura, K.; Kakutani, N.; Sudo, M.; Chemosphere 2014, 110, 78.

108. Poma, G.; Volta, P.; Roscioli, C.; Bettinetti, R.; Guzzella, L.; Sci. Total Environ. 2014, 481, 401.

109. Stapleton, H. M.; Dodder, N. G.; Kucklick, J. R.; Reddy, C. M.; Schantz, M. M.; Becker, P. R.; Gulland, F.; Porter, B. J.; Wise, S. A.; Mar. Pollut. Bull. 2006, 52, 522.

110. Ueno, D.; Alaee, M.; Marvin, C.; Muir, D. C. G.; Macinnis, G.; Reiner, E.; Crozier, P.; Furdui, V. I.; Subramanian, A.; Fillmann, G.; Lam, P. K. S.; Zheng, G. J.; Muchtar, M.; Razak, H.; Prudente, M.; Chung, K.; Tanabe, S.; Environ. Pollut. 2006, 144, 238.

111. Vorkamp, K.; Bossi, R.; Bester, K.; Bollmann, U. E.; Boutrup, S.; Sci. Total Environ. 2014, 470-471, 459.

112. Pizzini, S.; Marchiori, E.; Piazza, R.; Cozzi, G.; Barbante, C.; Microchem. J. 2015, 121, 184.

113. Zhu, N.; Schramm, K.-W.; Wang, T.; Henkelmann, B.; Fu, J.; Gao, Y.; Wang, Y.; Jiang, G.; Sci. Total Environ. 2015, 518-519, 328.

114. Janák, K.; Covaci, A.; Bechert, G.; Environ. Sci. Technol. 2005, 39, 1987.

115. Ramu, K.; Kajiwara, N.; Isobe, T.; Takahashi, S.; Kim, E. Y.; Min, B. Y.; We, S-U.; Tanabe, S.; Environ. Pollut. 2007, 148, 562.

116. Du, M.; Lin, L.; Yan, C.; Zhang, X.; Environ. Sci. Technol. 2012, 46, 11040.

117. Ibhazehiebo, K.; Iwasaki, T.; Xu, M.; Shimokawa, N.; Koibuchi, N.; Neurosci. Lett. 2011, 493, 1.

118. Marteinson, S. C.; Bird, D. M.; Letcher, R. J.; Sullivan, K. M.; Ritchie, I. J.; Fernie, K. J.; Chemosphere 2012, 89, 1077.

119. Du, M.; Zhang, D.; Yan, C.; Zhang, X.; Aquat. Toxicol. 2012, 112-113, 1.

120. http://chm.pops.int/TheConvention/POPsReviewCommittee/ Reports/tabid/2301/ctl/Download/mid/7538/Default. aspx $? \mathrm{id}=341 \& \mathrm{ObjID}=11423$, acessada em Setembro 2016.

121. Eljarrat, E.; Barceló, D. Em Brominated Flame Retardants; Eljarrat, E.; Barceló, D., eds.; Springer: Berlin, 2011.

122. Leonel, J.; Sericano, J. L.; Secchi, E. R.; Bertozzi, C.; Fillmann, G.; Montone, R. C.; Sci. Total Environ. 2014, 493, 405.

123. Leonel, J.; Sericano, J. L.; Fillmann, G.; Secchi, E.; Montone, R. C.; Mar. Pollut. Bull. 2010, 60, 412.
124. Quinete, N.; Lavandier, R.; Dias, P.; Taniguchi, S.; Montone, R.; Moreira, I.; Mar. Pollut. Bull. 2011, 62, 440.

125. Silva, S. F. G.; Tese de Doutorado, Pontifícia Universidade Católica, Brasil, 2009.

126. Cascaes, M. J.; Oliveira, R. T.; Ubarana, M. M.; Sato, R. M.; Baldassin, P.; Colabuono, F. I.; Leonel, J.; Taniguchi, S.; Weber, R. R.; Mar. Pollut. Bull. 2014, 86, 591.

127. Souza, A. C.; Monografia, Universidade Federal do Paraná, Brasil, 2015.

128. Kalantzi, O. I.; Brown, F. R.; Caleffi, M.; Goth-Goldstein, R.; Petreas, M.; Environ. Int. 2009, 35, 113.

129. Dorneles, P. R.; Lailson-Brito, J.; Dirtu, A. C.; Weijs, L.; Azevedo, A. F.; Torres, J. P. M.; Malm, O.; Neels, H.;Blust, R.; Das, K.; Covaci, A.; Environ. Int. 2010, 36, 60.

130. Leonel, J.; Taniguchi, S.; Sasaki, D. K.; Cascaes, M. J.; Dias, P. S.; Botta, S.; Santos, M. C. O.; Montone, R. C.; Chemosphere 2012, 86, 741.

131. Fillmann, G.; Leonel, J. Em Global Contamination Trends of Persistent Organic Chemical; Loganathan, B. G.; Lam, P. K. S., eds.; Taylor and Francis Group: United Kingdom, 2011, cap.18.

132. Baldassin, P.; Taniguchi, S.; Gallo, H.; Maranho, A.; Kolesnikovas, C.; Amorim, D. B.; Mansilla, M.; Navarro, R. M.; Tabeira, L. C.; Bicego, M. C.; Montone, R. C.; Chemosphere 2016, 149, 391.

133. Magalhães, C.; Taniguchi, S.; Cascaes, M. J.; Montone, R. C.; Mar. Pollut. Bull. 2012, 64, 662.

134. Ueno, D.; Kajiwara, N.; Tanaka, H.; Subramanian, A.; Fillmann, G.; Lam, P. K. S.; Zheng, G. J.; Muchitar, M.; Razak, H.; Prudente, M.; Chung, K.; Tanabe, S.; Environ. Sci. Technol. 2004, 38, 2312.

135. Pieroni, M. C.; Dissertação de Mestrado, Universidade Federal do Rio Grande, Brasil, 2012

136. Merico, A.; Montone, R. C.; Silva, J.; Seixas, T. G.; Godoy, J. M.; Saint'Pierre, T. D.; Hauser-Davis, R. A.; Di Beneditto, A. P.; Reis, E. C.; Tavares, D. C.; Lemos, L. S.; Siciliano, S.; Mar. Pollut. Bull. 2015, 95, 402.

137. Lavandier, R.; Arêas, J.; Quinete, N.; de Moura, J. F.; Taniguchi, S.; Montone, R.; Siciliano, S.; Moreira, I.; Environ. Pollut. 2015, 208, 442.

138. Pegoraro, C. N.; Harner, T., Su, K.; Chiappero, M. S.; Sci. Total Environ. 2016, 571, 172.

139. Lee, S. C.; Sverko, E.; Harner, T.; Pozo, K.; Barresi, E.; Schachtschneider, J.; Zaruk, D.; DeJong, M.; Narayanet, J.; Environ. Pollut. 2016, Article in press. http://dx.doi.org/10.1016/j.envpol.2016.01.080

140. Waaijers, S. L.; Kong, D.; Hendriks, H. S.; de Wit, C. A.; Cousins, I. T.; Westerink, R. H. S.; Leonards, P. E. G.; Kraak, M. H. S.; Admiraal, W.; de Voogt, P.; Parsons, J. R. Em Reviews of Environmental Contamination and Toxicology; Whitacre, D. M. ed.; Springer: New York, 2013, Cap. 1.

141. Rauert, C.; Harner, T.; Schuster, J. K.; Quinto, K.; Fillmann, G.; Castillo, L. E.; Fentanes, O.; Ibarra, M. V.; Miglioranza, K. S.; Rivadeneira, I. M.; Pozo, K.; Puerta, A. P.; Zuluaga, B. H. A.; Sci. Total Environ. 2016, Article in press. doi: 10.1016/j.scitotenv.2016.06.229

142. Law, R. J.; Herzke, D.; Em Brominated Flame Retardants; Eljarrat, E., Barceló, D., eds.; Springer: Berlin, 2011, Cap. 5. 